\title{
Inseguridad escolar y problemas académicos en una universidad pública mexicana
}

\author{
School insecurity and academic failure in a Mexican public university \\ Juan Manuel Hernández Vázquez \\ Javier Rodríguez Lagunas \\ Marco Antonio Leyva Piña
}

\begin{abstract}
RESUMEN
El objetivo de este artículo es ofrecer elementos para valorar los efectos que está teniendo la inseguridad, tanto interna como externa, en la actividad académica del alumnado universitario. Se exploran los casos de los campus Iztapalapa y Xochimilco de la Universidad Autónoma Metropolitana, establecida en la Ciudad de México. Se entiende que la seguridad física es un componente de la habitabilidad educativa de los espacios universitarios, que también contribuye a la calidad educativa. La investigación empírica se basó en una encuesta levantada durante los primeros meses del 2018, sobre muestras por cuotas de alumnos. Los resultados indican que, al parecer, Iztapalapa tiene más problemas de inseguridad que Xochimilco; que aproximadamente la mitad de los alumnos ha sido víctima de inseguridad al interior o en los alrededores del campus, y que, de las víctimas, casi la mitad ha padecido alguna consecuencia académica, porque al menos tuvo que faltar a clases o incluso tuvo que abandonar la institución. El hecho de que 10\% haya abandonado sus estudios por motivos de inseguridad podría representar una contribución importante al problema de la baja eficiencia terminal de la institución, la cual ronda $50 \%$, a los diez años del ingreso.
\end{abstract}

Palabras clave: ambiente de aprendizaje, habitabilidad educativa, enseñanza superior, punto de vista del alumnado, universidad pública.

\section{AbSTRACT}

The objective of this paper is to provide elements to assess the effects of internal as well external insecurity in the academic activity of university students. We explored the cases of the Iztapalapa and Xochimilco campuses of the Universidad Autónoma Metropolitana, located in Mexico City. Physical security is understood as a component of the educational habitability of university spaces, contributing to educational quality. The empirical research was based on a survey applied during the first months of 2018, by sampling the students in the two campuses. Amongst the findings, Iztapalapa appears to have more insecurity issues than Xochimilco; approximately half of the students have been victims of insecurity inside or around the campus, and almost half of them have suffered academic consequences such as missing classes or deserting the institution. The fact that $10 \%$ of the students have abandoned their studies due to insecurity could represent an important fact of low terminal efficiency, which is around $50 \%$ ten years after admission.

Keywords: learning environment, educational habitability, higher education, student's point of view, public university.

Recibido: septiembre 24 de 2020 | Aprobado: abril 27 de 2021 | Publicado: mayo 22 de 2021

DOI: https://doi.org/10.33010/ie_rie_rediech.v12i0.1114 


\section{INTRODUCCIÓN}

Frecuentemente, al hablar de la calidad de la educación generada en los espacios universitarios, se piensa casi exclusivamente en los aprendizajes que los estudiantes alcanzan mientras permanecen afiliados a la institución, pero es posible sostener que la calidad educativa universitaria también tiene que ver con la existencia de ambientes educativamente habitables, que propicien la ocurrencia de experiencias positivas de aprendizaje. La literatura especializada sugiere que los objetivos escolares deben ser revisados para incluir no solo los aprendizajes académicos sino también la generación de ambientes físicos e interaccionales saludables (Thapa, Cohen, Guffey y HigginsD’Alessandro, 2013; Cohen, 2006).

De acuerdo con estas ideas, la habitabilidad educativa universitaria tiene que ver con qué tanto las universidades disponen de espacios educativos con ambientes de alojamiento y asentamiento propicios para dar lugar a procesos eficaces tanto de producción y de reproducción-apropiación del conocimiento como de preservación y difusión de la cultura, procesos que constituyen las actividades sustantivas de las universidades. En el caso de la Universidad Autónoma Metropolitana (UAM), estas actividades quedaron consagradas en la ley orgánica que le dio origen en 1973.

Un concepto operativo de habitabilidad educativa universitaria puede basarse en la idea planteada por Hernández (2010) para el caso de la educación básica, en donde la seguridad física aparece como una de sus diez dimensiones centrales (figura 1).

Importa considerar que la seguridad física, lo mismo que su opuesta, la inseguridad física, involucra aspectos asociados tanto al diseño y mantenimiento de las instalaciones como también a la clase de relaciones que se pueden dar entre quienes ingresan a las mismas o se mueven en sus periferias. Esta idea se encuentra estrechamente vinculada con lo que se ha concebido como seguridad escolar (Butkus, 2020;

Juan Manuel Hernández Vázquez. Profesor-investigador del Departamento de Sociología en la Universidad Autónoma Metropolitana- Iztapalapa, Ciudad de México. Es doctor en Estudios Sociales por la misma universidad y miembro del Sistema Nacional de Investigadores. Entre sus publicaciones recientes se encuentran los artículos "La multidisciplina en los estudios universitarios. La perspectiva de los alumnos de la UAM- Iztapalapa" (2020) y "El trabajo de la juventud NiNi en los hogares mexicanos” (2020). Correo electrónico: jm.uami@gmail.com. ID: https://orcid.org/0000-0002-6009-5545.

Javier Rodríguez Lagunas. Profesor-investigador del Departamento de Sociología en la Universidad Autónoma Metropolitana- Iztapalapa, Ciudad de México. Doctor en Sociología por la Universidad Nacional Autónoma de México. Miembro del Sistema Nacional de Investigadores, nivel I. Entre sus publicaciones recientes se encuentran el libro Los académicos mexicanos ante las reformas universitarias y el artículo "La reforma de la educación superior en México, entre la espada y la pared. La mirada de los universitarios”. Correo electrónico: rolj@xanum.uam.mx. ID: https://orcid.org/0000-0002-1527-1490.

Marco Antonio Leyva Piña. Profesor-investigador del Departamento de Sociología en la Universidad Autónoma Metropolitana- Iztapalapa, Ciudad de México. Doctor en Ciencias Sociales por la Universidad Autónoma Metropolitana- Xochimilco, Ciudad de México. Entre sus publicaciones recientes se encuentra el artículo "La reforma de la educación superior en México, entre la espada y la pared. La mirada de los universitarios”. Correo electrónico: marcolp2000@yahoo.com.mx. ID: https:// orcid.org/0000-0001-5642-9410. 


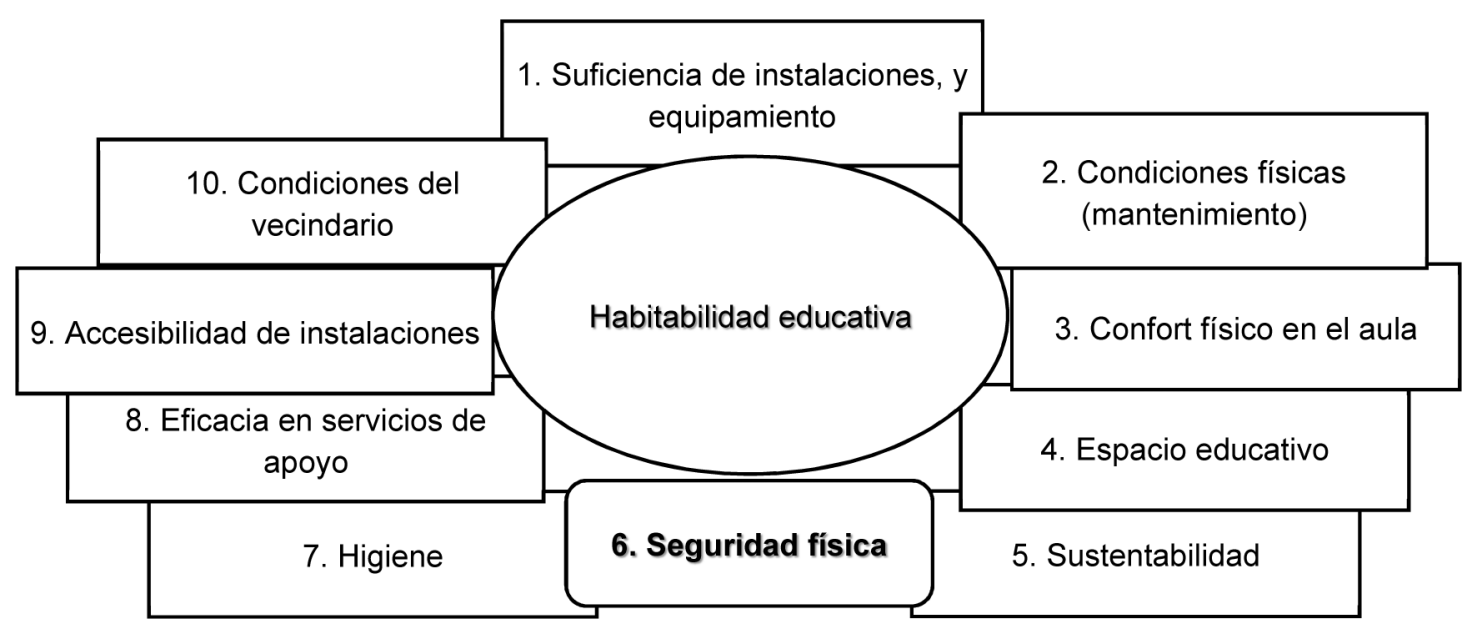

Figura 1. Dimensiones de la habitabilidad educativa.

Fuente: Construcción personal con base en Hernández, 2010.

Tanner-Smith, Fisher, Addington y Gardella, 2017). Si bien los universitarios necesitan sentir, por ejemplo, que no les caerá encima un plafón o una barda y que no caerán de bruces cuando transiten por un pasillo, también necesitan certeza de que no serán golpeados y despojados de sus pertenencias cuando transitan por la calle que los conduce al campus, lo mismo que durante su estancia en el interior del mismo. Es este componente interaccional de la seguridad física el que ha sido enfocado por este trabajo de investigación.

Buena parte de la investigación sobre seguridad física ha sido dedicada a observar problemáticas relacionadas con la construcción de ambientes educativos resultantes de la interacción del alumnado con los espacios físicos escolares, pero solo recientemente se ha puesto atención en cuestiones relativas a las interacciones de las personas habitantes o visitantes de dichos espacios.

La abultada literatura sobre Crime Prevention Through Environmental Design (CPTED) enfatiza cómo el diseño de los espacios puede reducir el riesgo y elevar la sensación de seguridad con áreas bien delimitadas y diseñadas para el control natural del acceso y para el reforzamiento de la vigilancia colectiva (Deutsch, 2019; Edmonton Police Service, 2018; Kennedy, 2017; Sohn, 2015). Estas ideas descansan en el entendido de que la construcción de ambientes educativamente habitables es resultado, en buena medida, de las decisiones sobre el diseño y mantenimiento de las instalaciones escolares, las cuales deben garantizar la integridad física de las personas en su interior, mediante 1) la disposición de medios físicos para escapar en caso de alguna emergencia o desastre natural (incendio, sismo, maremoto, inundación), 2) la previsión de las acciones a realizar en caso de ocurrir algún siniestro y 3) el establecimiento de medidas de seguridad en auditorios, laboratorios, talleres, cocinas, hornos, interruptores de energía, entre otros. 
Asimismo, diversos estudios han enfatizado las evidencias de que si las instalaciones escolares son inseguras los alumnos tendrán desventajas en el aprendizaje (Erichsen, 2020; Fritz, 2003). Por ello, investigadores como Côté-Lussier y Fitzpatrick (2016) proponen a las escuelas la realización de intervenciones para mejorar la sensación de seguridad entre sus habitantes, porque encontraron que esta se asocia a menores síntomas de depresión, lo cual mejora la atención y el compromiso en el aula, y posiblemente también contribuya a elevar el logro académico y la permanencia, lo mismo que a mitigar los problemas de salud mental en el alumnado.

Otra cuestión a tomar en cuenta es que la seguridad física de los usuarios de los espacios escolares involucra dos componentes, uno interno y otro externo, porque la generación y preservación de ambientes físicos educativamente habitables no solo resulta de las acciones de los gobiernos internos universitarios sino también de las decisiones eficaces de gobernanza (Ostrom, 2014), tanto en el ámbito local como en el federal, sobre los entornos donde se asientan los recintos universitarios. Dubet (2003) resalta esta conexión entre los ámbitos intra y extraescolar cuando señala la existencia de vasos comunicantes entre el ambiente escolar y el social, el interno y el externo a la escuela.

Existe una especie de frontera difusa escolar de la que ya ha dado cuenta la investigación educativa. Baste mencionar el trabajo de Ramos y Vázquez (2013) sobre el consumo de drogas ilícitas en el recinto escolar, y el de González, Inzunsa y Benítez (2013) sobre la recién evidenciada penetración de la narcoviolencia en las comunidades escolares.

El presente documento buscar aportar elementos para responder dos preguntas torales: ¿En qué medida ocurren acciones delictivas, tanto al interior como en los alrededores del campus, dirigidas a los alumnos? y ¿Qué tanto perturban su logro académico? Es importante prestar atención a este objeto de estudio, hasta ahora prácticamente inobservado, a pesar de su evidente presencia, dado que la inquietud investigativa ha sido orientada fundamentalmente a otras temáticas de seguridad y violencia escolar, como el acoso, el ciberbullying o la narcoviolencia.

Se entiende que el análisis de la perspectiva estudiantil es importante para la comprensión del fenómeno de la inseguridad universitaria, en un contexto en el que la ocurrencia de actos delictivos, tanto al interior como al exterior de las universidades, parece tomar dimensiones alarmantes. Cada vez con mayor frecuencia ocurren situaciones en las que son comprometidas tanto la integridad física de los universitarios como la propiedad de sus bienes.

Un seguimiento a las publicaciones del grupo de Facebook UAM Iztapalapa, realizado entre el 15 de febrero y el 15 de diciembre del año 2017 registró 28 publicaciones realizadas por alumnos, a través de las cuales advertían a sus compañeros sobre la ocurrencia de diversos problemas de seguridad interna y externa: asaltos en 
las salidas del campus, en sus alrededores y en los transportes locales; robos al interior del campus, perpetrados por personas ajenas a la comunidad universitaria, como el de la nómina de trabajadores en la cafetería, y hurtos de bicicletas, laptops y diversas pertenencias resguardadas en los automóviles estacionados en espacios interiores. Varios mensajes también fueron aprovechados para enviar mensajes de auxilio a las autoridades, quejarse del personal encargado de la vigilancia interna y convocar a formar grupos estudiantiles de autodefensa.

Diversas acciones de las autoridades universitarias también dan cuenta del estado de emergencia reciente en el campus Iztapalapa de la UAM (UAMI). Particularmente notorias han sido la emisión de varias comunicaciones institucionales, distribuidas vía correo electrónico, con la intención de advertir sobre hurtos, robos y asaltos dirigidos a la comunidad universitaria en varios lugares del campus y en sus alrededores, y la formación de una comisión de seguridad interna para idear acciones tendientes a enfrentar estos problemas (UAMI, 2017a, 2017b y 2017c).

\section{SEGURIDAD FÍSICA INTERNA Y EXTERNA}

Una placa con una dedicatoria póstuma, fijada en una columna interior frente a la entrada del edificio H de la UAMI, recuerda las emociones de inseguridad y de dolor vividas por la comunidad universitaria el 28 de febrero del 2012, al enterarse de que uno de sus profesores más reconocidos había sido asesinado, justo a la entrada principal del campus, solo por resistirse a los amagos de un desconocido que intentó arrebatarle un dinero apenas retirado de una cuenta bancaria (Notimex, 2012; Redacción Proceso, 2012).

Aquel acontecimiento, que incluso tuvo resonancia nacional, refuerza la idea de que los usuarios de las instalaciones universitarias necesitan afianzar su seguridad en que su integridad física, lo mismo que la propiedad de sus pertenencias, no se verán afectadas mientras desarrollan actividades académicas intramuros o al desplazarse por los alrededores. Esta clase de seguridad se relaciona directamente con la habitabilidad educativa del campus, en tanto que involucra la generación y sostenimiento de ambientes seguros y saludables, tanto en el interior como en el entorno del campus.

La construcción de la seguridad física de corte interaccional requiere gobernanzas fuertes (Mayorga y Córdova, 2007), por las que tanto la universidad como los gobiernos local y federal, cada uno en sus ámbitos de acción, tomen decisiones y emprendan acciones para la eficaz prevención, control y supresión de actos delictivos. La figura 2 sugiere que la dimensión de seguridad física requiere gobernanzas referidas tanto a gestión y diseño de los espacios físicos escolares como a las condiciones del vecindario en que se asientan dichos espacios.

Existen conexiones claras entre la escuela y su entorno, entre la inseguridad interior y la exterior. Dubet (2003) resalta que la crisis generalizada (económica, política 


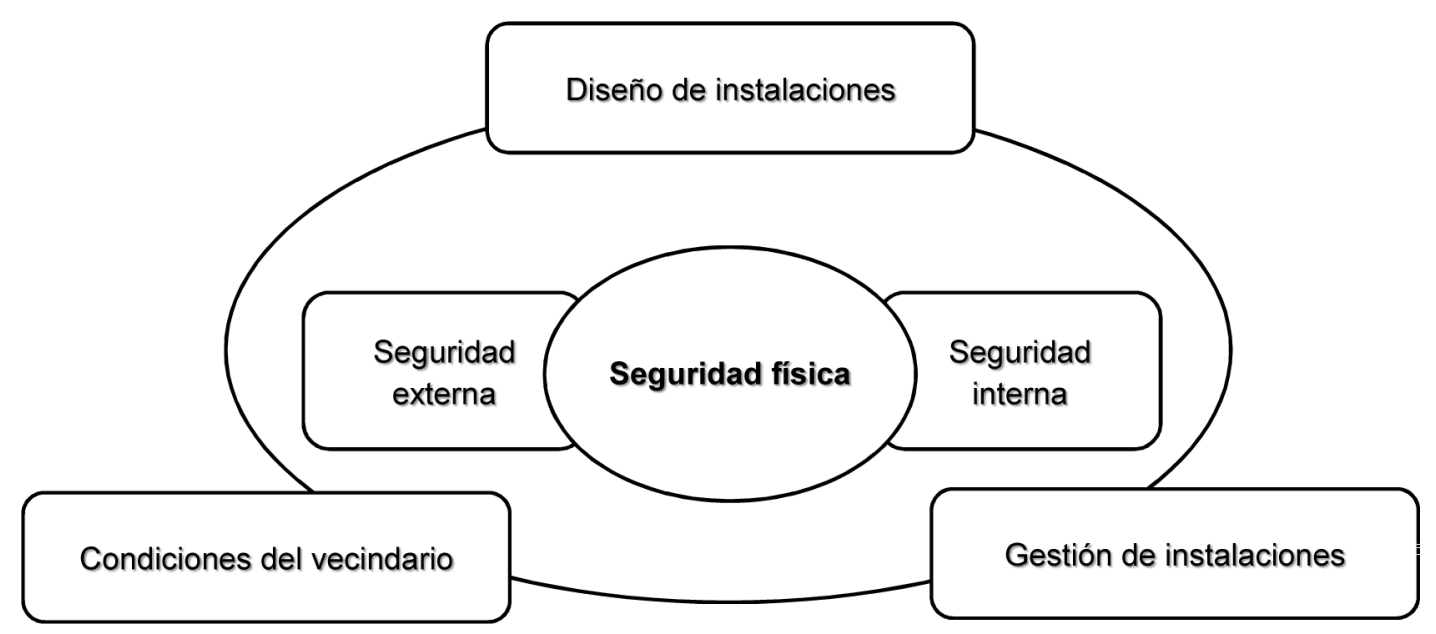

Figura 2. Componentes de la seguridad física.

Fuente: Construcción personal.

y social) impulsa el desbordamiento de la delincuencia y la violencia de todo tipo en los alrededores, y que esta violencia finalmente termina por invadir la escuela por dos razones: porque los procesos de masificación educativa han abierto el acceso a grupos poblacionales antes excluidos, y porque se ha debilitado el papel de la educación como mecanismo de ascenso social. La investigación educativa ya ha dado cuenta de este fenómeno que desvanece la frontera escolar.

En su revisión de temáticas actuales, Fierro (2017) resalta investigaciones sobre el aumento en el consumo de drogas ilícitas en los recintos escolares (Ramos y Vázquez, 2013) y la recién evidenciada penetración de la narcoviolencia en las comunidades escolares (González, Inzunsa y Benítez, 2013), penetración manifiesta en las extorsiones, secuestros, robos y asaltos dirigidos principalmente al magisterio, y en la objetivación de identidades estudiantiles que exaltan y rinden culto al narcotráfico, simbolizando así el cambio en los mecanismos de aspiraciones de ascenso social. En centros educativos de puertas abiertas, como la UAMI, estos vasos comunicantes pudieran ser particularmente fluidos debido al escaso control del acceso a personas ajenas a la comunidad universitaria.

No se sabe a ciencia cierta qué tanto ocurren actos delictivos al interior de las instituciones de educación superior (IES), en un contexto en el que los indicadores muestran que, desde el 2007, aumentan constantemente los actos homicidas a nivel nacional (UNODC, 2019). No obstante, es encomiable la iniciativa de la ANUIES porque evidenció el problema de la inseguridad al interior de las IES con la publicación de un manual de seguridad (ANUIES, 2011) y un diagnóstico de inseguridad que incluye recomendaciones (Garnica, 2012), iniciativa a la que universidades importantes, como la UNAM y la UAM, no se han adherido porque, entre otras medidas, la prueba toxicológica a estudiantes es considerada como "violatoria de los derechos humanos" 
(Poy, 2012). El diagnóstico establece, como referente de evaluación, un sistema de seguridad compuesto por cinco componentes: 1) establecimiento de normas, 2) idear un plan de prevención, 3) idear protocolos de atención de incidentes, 4) creación de un comité o consejo de seguridad, 5) disponer de recursos humanos, equipo e infraestructura de seguridad, y dimensiona hasta qué punto las IES cuentan con cada uno de ellos. También exhibe un listado de los principales incidentes delictivos que ocurren al interior de las IES, basado en información cualitativa, aunque no precisa la frecuencia con que se presenta cada uno de ellos. Seguimos sin saber qué tanto ocurren actos delictivos al interior de las universidades, debido al hermetismo de sus directivos; sin embargo, el documento es de gran valía porque resalta la importancia de tomar en cuenta la zona de asentamiento, donde ocurren actos delictivos que "están afectando directamente a los estudiantes universitarios" (Garnica, 2012, p. 123), como el asalto a transeúntes, asalto en transporte público, extorsión, agresión sexual y venta de estupefacientes y de bebidas alcohólicas.

Se aprecia, por la frecuencia con que diversas fuentes noticiosas dan cuenta de ello, que los problemas de inseguridad escolar en nuestro país van en aumento (Marván, 2018), lo mismo que sus efectos sobre la actividad académica de los estudiantes involucrados (Imagen Radio, 2020; Dávila, 2018; Villalobos, 2018; Cedillo, 2015). No obstante, la investigación educativa aún ignora las dimensiones de tales efectos, y tampoco se ha inquietado por saber qué tanto ocurren actos delictivos en los centros educativos universitarios y en sus alrededores. Es necesario, pues, dedicar energías investigativas para saber no solo cuál es la prevalencia del fenómeno sino también para valorar cuáles son sus consecuencias académicas, como dejar de asistir a clases, bajar de calificaciones, reprobar asignaturas o, en el caso extremo, abandonar temporal o definitivamente los estudios universitarios. Esta investigación busca aproximaciones en ambas direcciones, estableciendo contrastes entre las dos unidades académicas sujetas a estudio, Iztapalapa y Xochimilco.

\section{Estrategia METOdológica}

Los análisis expuestos más adelante corresponden a una investigación exploratoria basada en la información captada durante la primera quincena de febrero del 2018, mediante un instrumento de encuesta cuyo cuerpo quedó conformado por cuatro secciones: la primera incluyó una batería de preguntas tipo Likert, mediante la cual se captó la frecuencia con que, según los estudiantes, ocurren dentro de las instalaciones universitarias actos que constituyen delitos (asalto, robo, abuso sexual, etc.) o transgresiones al reglamento de alumnos (consumo de embriagantes, portar armas, etc.); la segunda sección, también diseñada en forma de escala Likert, permitió saber si el propio informante o algún compañero había sido víctima de actos delictivos al interior del campus o en las inmediaciones; la tercera captó información sobre 
las consecuencias académicas de dichos actos (ausentismo, reprobación, abandono, etc.); finalmente, la última sección del instrumento permitió saber cuántos establecimientos identifican los alumnos que se encuentran en los alrededores del campus, a pesar de no estar permitidos por la normatividad de la Ciudad de México, y captar las sugerencias del alumnado para mejorar las condiciones de seguridad universitaria.

El levantamiento de la información operó con base en un diseño muestral por cuotas de 174 y 170 estudiantes, pertenecientes a los campus Iztapalapa y Xochimilco de la UAM (UAMI y UAMX), respectivamente. Se cuidó que estuvieran representados, proporcionalmente, los hombres y las mujeres formalmente inscritos como alumnos al momento de la aplicación en cada una de las carreras impartidas en estas unidades académicas. Con ello fue posible explorar contrastes entre las mismas. Se avanzó en probar la conjetura de que la UAMI podría ser, de las dos unidades analizadas, la más afectada por la inseguridad interna.

En la actualidad la UAM es una de las instituciones mexicanas de educación superior más reconocidas por sus resultados académicos; en algunos rankings internacionales supera incluso a las más prestigiadas instituciones públicas nacionales de educación superior, como la Universidad Nacional Autónoma de México y el Instituto Politécnico Nacional (Times Higher Education, 2019). Los campus Iztapalapa y Xochimilco son parte de los tres con que nació la institución en 1974 (actualmente también la conforman Azcapotzalco, Cuajimalpa y Lerma), y juntos captan la mayor parte de la matrícula. En el 2018 registraron 60\% de los 45,936 alumnos matriculados en la institución (UAM, 2019). Dentro de la UAM, el campus de Iztapalapa es el más acreditado por sus elevados estándares de trabajo académico (concentra 42\% de la plantilla académica de la UAM que es reconocida por el Sistema Nacional de Investigadores), y el de Xochimilco por su elevada eficiencia terminal (70\% de su alumnado concluye la licenciatura, cuando mucho, a los diez años de haber ingresado).

La organización académica de ambos campus es departamental, en contraste con la clásica estructura organizacional por facultades, pero sus modelos de enseñanza son fundamentalmente diferentes. En Iztapalapa los profesores imparten clases con el formato tradicional de exposiciones frente a los grupos, en correspondencia con asignaturas independientes. En cambio, en Xochimilco se sigue un sistema modular, por el que los docentes no se ajustan al típico papel de impartidores de clases, sino de asesores-coordinadores de investigaciones que son desarrolladas por los grupos de estudiantes. Al final, las calificaciones asentadas no son sobre asignaturas independientes sino sobre módulos temáticos integradores.

\section{Resultados. Las UAM Iztapalapa y Xochimilco en CONTRASTE}

¿Qué tanta inseguridad interna es percibida por los alumnos en cada campus, y cuáles problemas consideran que son los más frecuentes? De acuerdo con la tabla 1, la gran 
mayoría de los alumnos encuestados, de ambas unidades académicas, consideró que ha ocurrido algún problema de inseguridad al interior de las instalaciones. No obstante, los datos indican que se percibe más prevalencia de inseguridad interna en Iztapalapa $(96 \%)$ que en Xochimilco (85.9\%), por una diferencia de casi 10 puntos porcentuales.

Tabla 1. Porcentaje de alumnos que consideran que con frecuencia ${ }^{1}$ ocurren problemas de inseguridad dentro del campus, según tipo de problema, 2018.

\begin{tabular}{|c|c|c|c|c|c|c|}
\hline \multirow[b]{2}{*}{ Tipo de problema } & \multicolumn{3}{|c|}{ Iztapalapa } & \multicolumn{3}{|c|}{ Xochimilco } \\
\hline & Hombre & Mujer & Total & Hombre & Mujer & Total \\
\hline Asalto a mano armada & 66.3 & 69.4 & 67.8 & 25.0 & 28.7 & 27.1 \\
\hline Robo sin violencia & 61.8 & 62.4 & 62.1 & 30.3 & 38.3 & 34.7 \\
\hline$\overline{\text { Abuso sexual }}$ & 21.3 & 14.1 & 17.8 & 17.1 & 25.5 & 21.8 \\
\hline Violación & 7.9 & 7.1 & 7.5 & 7.9 & 11.7 & 10.0 \\
\hline Golpes entre universitarios & 29.2 & 17.6 & 23.6 & 22.4 & 27.7 & 25.3 \\
\hline Asesinato & 9.0 & 15.3 & 12.1 & 7.9 & 10.6 & 9.4 \\
\hline Consumo de alcohol & 76.4 & 75.3 & 75.9 & 73.7 & 66.0 & 69.4 \\
\hline Consumo de drogas ilícitas & 82.0 & 77.6 & 79.9 & 73.7 & 74.5 & 74.1 \\
\hline Tráfico de drogas & 64.0 & 42.4 & 53.4 & 55.3 & 55.3 & 55.3 \\
\hline Portación de armas de fuego & 14.6 & 10.6 & 12.6 & 4.0 & 10.6 & 7.7 \\
\hline Entran personas de fuera para cometer delitos & 83.1 & 75.3 & 79.3 & 31.6 & 42.6 & 37.6 \\
\hline Alguno de los anteriores & 94.4 & 97.6 & 96.0 & 85.5 & 86.2 & 85.9 \\
\hline
\end{tabular}

1 Porcentaje de alumnos que contestaron "muy frecuente" o "algo frecuente".

Fuente: Construcción personal con base en la Encuesta sobre seguridad e bigiene desde la perspectiva de los alumnos de la UAM, 2018.

Según los alumnos de ambos campus, entre los actos delictivos más frecuentes ocurridos al interior están el consumo de alcohol y de drogas ilícitas, lo mismo que el tráfico de drogas. Más del 50\% observa que esta clase de problemas de inseguridad ocurre dentro de las instalaciones. No obstante, sobre todo en Iztapalapa, la situación es particularmente grave debido a la percepción de que más frecuentemente entran personas extrañas para cometer delitos y ocurren actos de robo con violencia, lo mismo que de asalto a mano armada. De hecho, estos tres aspectos de inseguridad interna registraron las mayores diferencias respecto a lo registrado en Xochimilco, entre 27 y 42 puntos porcentuales. La adición de estos tres problemas indica una particular oportunidad para la mejora en la gobernanza interna de la UAMI, específicamente en lo tocante a las decisiones que se toman sobre el control de los accesos, decisiones que son de crucial importancia para mejorar las condiciones de seguridad al interior y, por lo mismo, para la construcción de ambientes seguros de aprendizaje. Por ello, la abultada literatura sobre el CPTED ha insistido en los detalles que conviene tomar en cuenta a la hora de diseñar las entradas a las instalaciones escolares (Deutsch, 2019; Edmonton Police Service, 2018; Kennedy, 2017; Sohn, 2015). 
Por su parte, en la UAMX los aspectos particularmente delicados parecen estar en el tráfico de drogas, los golpes entre universitarios y los temas de agresión sexual. En estos tres aspectos las diferencias con Iztapalapa rondan los 2 o 4 puntos porcentuales. Aunque esta diferencia es pequeña, conviene que sea tomada en cuenta. Una estimación más precisa ameritaría invertir más recursos en la implementación de alguna técnica de muestreo probabilístico.

Finalmente, los datos aportados por los encuestados indican que por lo general las alumnas de ambos campus, en comparación con los hombres, consideran que ocurren ligeramente más problemas de inseguridad. Específicamente en Iztapalapa ellas son relativamente más impresionadas que los hombres por los eventos de asesinato, mientras que en Xochimilco, por la entrada de delincuentes al campus, el robo sin violencia, la portación de armas de fuego y las agresiones sexuales.

Esta es la apreciación del alumnado, pero, ¿cuál es la prevalencia real de la inseguridad, interna y externa? La información de la tabla 2 ayuda a contestar esta pregunta. Muestra que, de acuerdo con la información proporcionada por los alumnos, alrededor de la mitad ha sido víctima de algún acto delictivo dentro o fuera del campus, quizás solo muy poco más en Iztapalapa que en Xochimilco $50 \%$ versus 48.2\%). Específicamente, en Iztapalapa la prevalencia de la inseguridad interna parece superar un poco a la de Xochimilco (27\% versus $20 \%$ ), pero en seguridad externa, ambos campus se parecen (42.5\% versus 40.6\%). No sorprende esta diferencia en inseguridad interna porque Iztapalapa no tiene control sobre sus accesos: cualquier

Tabla 2. Porcentaje de alumnos que han sufrido ${ }^{1}$ algún problema de inseguridad, según lugar donde lo sufrieron, 2018.

\begin{tabular}{|c|c|c|c|}
\hline \multirow[b]{2}{*}{ Campus } & \multicolumn{2}{|c|}{ Sexo } & \multirow[b]{2}{*}{ Total } \\
\hline & Hombre & Mujer & \\
\hline \multicolumn{4}{|c|}{ Dentro del campus } \\
\hline Iztapalapa & 25.8 & 28.2 & 27.0 \\
\hline Xochimilco & 17.1 & 22.3 & 20.0 \\
\hline Total & 21.8 & 25.1 & 23.5 \\
\hline \multicolumn{4}{|c|}{ En los alrededores del campus } \\
\hline Iztapalapa & 39.3 & 45.9 & 42.5 \\
\hline Xochimilco & 35.5 & 44.7 & 40.6 \\
\hline Total & 37.6 & 45.3 & 41.6 \\
\hline \multicolumn{4}{|c|}{ Dentro o en los alrededores } \\
\hline Iztapalapa & 46.1 & 54.1 & 50.0 \\
\hline Xochimilco & 44.7 & 51.1 & 48.2 \\
\hline Total & 45.5 & 52.5 & 49.1 \\
\hline
\end{tabular}

Fuente: Construcción personal con base en la Encuesta sobre seguridad e bigiene desde la perspectiva de los alumnos de la UAM, 2018. 
persona extraña puede entrar y salir a discreción. Tampoco sorprende el parecido en cuanto a la inseguridad en los alrededores, porque el campus Xochimilco, si bien se asienta en la alcaldía de Coyoacán, se encuentra casi en la colindancia con las alcaldías de Iztapalapa, entre las más violentas, y de Xochimilco. Los datos también indican que las mujeres tienden a ser frecuentemente más victimizadas que los hombres, tanto en el interior como en el exterior.

¿Cuáles son los tipos de delitos que sufren más los universitarios en el campus? Los datos desagregados en la tabla 3, por tipo de acto delictivo realizado en contra de los alumnos encuestados, confirman la idea de que en Iztapalapa la inseguridad interna es más prevaleciente en prácticamente todas las modalidades consideradas, excepto las relacionadas con la violencia sexual. Por ejemplo, las más importantes: el hurto y el intento de robo registraron en Iztapalapa 23\% y $9.2 \%$, mientras que en Xochimilco, $16.5 \%$ y 6.5\%. Nuevamente sobresale que en este campus ocurren relativamente más problemas de violencia sexual $(7 \%$, juntando violación y abuso sexual), en contraste con Iztapalapa (2.3\%), posiblemente debido a la forma como fueron distribuidos los edificios en el terreno.

Tabla 3. Porcentaje de alumnos que han sufrido algún problema de inseguridad, según lugar donde lo sufrieron y tipo de problema, 2018.

\begin{tabular}{|c|c|c|c|c|c|c|}
\hline \multirow[b]{2}{*}{ Tipo de problema sufrido } & \multicolumn{3}{|c|}{ Iztapalapa } & \multicolumn{3}{|c|}{ Xochimilco } \\
\hline & Hombre & Mujer & Total & Hombre & Mujer & Total \\
\hline \multicolumn{7}{|c|}{ Dentro del campus $^{1}$} \\
\hline Hurto & 21.3 & 24.7 & 23.0 & 13.2 & 19.1 & 16.5 \\
\hline Intento de Robo & 7.9 & 10.6 & 9.2 & 2.6 & 9.6 & 6.5 \\
\hline Robo con violencia & 2.2 & 5.9 & 4.0 & 3.9 & 4.3 & 4.1 \\
\hline Robo a mano armada & 3.4 & 3.5 & 3.4 & 0.0 & 3.2 & 1.8 \\
\hline Abuso sexual & 0.0 & 1.2 & 0.6 & 1.3 & 5.3 & 3.5 \\
\hline Violación & 2.2 & 1.2 & 1.7 & 2.6 & 4.3 & 3.5 \\
\hline \multicolumn{7}{|c|}{ En los alrededores del campus ${ }^{2}$} \\
\hline Hurto & 20.2 & 24.7 & 22.4 & 22.4 & 26.6 & 24.7 \\
\hline Intento de Robo & 25.8 & 31.8 & 28.7 & 25.0 & 26.6 & 25.9 \\
\hline Robo con violencia & 24.7 & 23.5 & 24.1 & 17.1 & 22.3 & 20.0 \\
\hline Robo a mano armada & 23.6 & 16.5 & 20.1 & 17.1 & 23.4 & 20.6 \\
\hline Abuso sexual & 7.9 & 12.9 & 10.3 & 5.3 & 7.4 & 6.5 \\
\hline Violación & 4.5 & 7.1 & 5.7 & 5.3 & 7.4 & 6.5 \\
\hline
\end{tabular}

1 Porcentaje respecto a los que contestaron que "sí" han sufrido algún problema de inseguridad dentro del campus.

2 Porcentaje respecto a los que contestaron que "sí" han sufrido algún problema de inseguridad en los alrededores.

Fuente: Construcción personal con base en la Encuesta sobre seguridad e higiene desde la perspectiva de los alumnos de la UAM, 2018. 
Estas diferencias entre los campus se reducen cuando se observa lo que ocurre en su entorno inmediato, pero posiblemente el de Iztapalapa sea solo ligeramente más inseguro. En ambos campus el intento de robo es el más importante $28.7 \%$ en Iztapalapa versus $25.9 \%$ en Xochimilco), seguido por robo con violencia, hurto y robo a mano armada (entre $20 \%$ y $25 \%$ en ambas unidades) y al final los problemas de violencia sexual (16\% en Iztapalapa y $13 \%$ en Xochimilco).

¿Qué tanto afecta la inseguridad al logro académico de los alumnos? Cuando los alumnos son víctimas de algún acto delictivo, al interior o en las cercanías del campus, su actividad académica se ve afectada al menos por tener que faltar a clases para realizar los trámites de denuncia ante las autoridades universitarias y/o ante el ministerio público de la alcaldía, pero, en circunstancias extremas, el impacto podría derivar en afectaciones físicas o psicológicas severas que impedirían definitivamente su permanencia en la universidad.

Los cálculos mostrados en la tabla 4 indican que, de acuerdo con las estimaciones del alumnado encuestado, una mayor proporción de víctimas en Iztapalapa sufre alguna de las consecuencias académicas estudiadas (58.6\%) en comparación con Xochimilco (48.8\%). Dichos cálculos también muestran que si bien en ambos campus la consecuencia más frecuente es simplemente faltar a clases $(40.2 \%$ en Iztapalapa y $37.1 \%$ en Xochimilco), el resto se ordena con un patrón diferenciado. En Iztapalapa siguen en orden de importancia dar de baja o renunciar a alguna asignatura (12.1\%), bajar de calificaciones y dejar de asistir a la institución (11.5\%) y al final reprobar alguna asignatura (6.3\%). En cambio, en Xochimilco siguen bajar de calificaciones (12.9\%), dejar de ir a la institución (8.8\%) y reprobar (7.6\%) o dar de baja algún módulo de asignaturas $(7.1 \%)$. Sorprende que dejar de ir a la UAM haya registrado porcentajes que parecen elevados. Es probable que la pregunta no haya captado abandono definitivo de los estudios. Esta es una cuestión para considerar en estudios futuros. De comprobarse las cifras, resultaría que solo el tema de la inseguridad (interna o

Tabla 4. Porcentaje de consecuencias académicas en las víctimas de inseguridad (dentro o fuera del campus), según tipo de consecuencia, 2018.

\begin{tabular}{|c|c|c|c|c|c|c|}
\hline \multirow[b]{2}{*}{ Tipo de consecuencia } & \multicolumn{3}{|c|}{ Iztapalapa } & \multicolumn{3}{|c|}{ Xochimilco } \\
\hline & Hombre & Mujer & Total & Hombre & Mujer & Total \\
\hline Faltar a clase & 38.2 & 42.4 & 40.2 & 36.8 & 37.2 & 37.1 \\
\hline Bajar de calificaciones & 14.6 & 8.2 & 11.5 & 11.8 & 13.8 & 12.9 \\
\hline Reprobar una o más asignaturas o el módulo & 7.9 & 4.7 & 6.3 & 6.6 & 8.5 & 7.6 \\
\hline Dar de baja o renunciar a una o más asignaturas, o al módulo & 13.5 & 10.6 & 12.1 & 7.9 & 6.4 & 7.1 \\
\hline Dejar de venir a la UAM & 13.5 & 9.4 & 11.5 & 10.5 & 7.4 & 8.8 \\
\hline Alguna de las anteriores & 58.4 & 58.8 & 58.6 & 50.0 & 47.9 & 48.8 \\
\hline
\end{tabular}

Fuente: Construcción personal con base en la Encuesta sobre seguridad e bigiene desde la perspectiva de los alumnos de la UAM, 2018. 
externa) abonaría en aproximadamente 5 puntos porcentuales al problema de la baja eficiencia terminal de la UAM, misma que ronda el 50\%, calculada diez años después del ingreso inicial de la generación correspondiente a la institución.

\section{DisCuSIÓN Y CONCLUSIONES}

Los resultados del estudio expuesto en este documento dieron cuenta de importantes oportunidades para mejorar la habitabilidad educativa referida a la seguridad física (interna y externa) en los campus Iztapalapa y Xochimilco de la UAM, sobre todo en el primero, desde la perspectiva del alumnado encuestado. Iztapalapa parece ser el campus que más requiere acciones decisivas de gobernabilidad para aliviar problemas de inseguridad interna. Como lo sugirió 61\% de los alumnos encuestados, la gobernabilidad interna mejoraría si se controlara la entrada de personas extrañas a las instalaciones. Una parte de esta clase de personas aprovecha el hecho de poder entrar y salir sin restricción ni registro alguno para perpetrar actos delictivos dirigidos a la comunidad universitaria, como el hurto y el robo. El hurto fue el más característico de Iztapalapa.

A pesar de que Xochimilco resultó en general menos insegura, al parecer sufre de relativamente más actos de violencia sexual que Iztapalapa. Es posible que la disposición de los edificios en aquel campus, confinando amplios jardines rectangulares, dificulte la vigilancia natural de los usuarios sobre algunas áreas particularmente inseguras. Los complejos constructivos de la UAMX, si bien benefician la generación de ambientes agradables por el contacto de los usuarios con la naturaleza verde, quizás al mismo tiempo no sean adecuados para la habitabilidad educativa relacionada con la seguridad interna, porque quedan zonas en su interior de tránsito peatonal lento, y vedadas a la vista vigilante de los usuarios, que pueden propiciar la comisión de actos delictivos asociados al tráfico de drogas, los golpes entre universitarios y las agresiones sexuales. Una importante proporción de alumnos sugirió el uso de modernas tecnologías de vigilancia para enfrentar esta clase de dificultades.

Ambos campus tienen problemas de inseguridad externa, pero, de acuerdo con las apreciaciones del alumnado, las difusas fronteras universitarias son transgredidas ligeramente más en el campus de Iztapalapa. No obstante, es posible que la situación esté cambiando positivamente por dos razones: primera, porque poco antes de empezar la pandemia por el COVID-19 la rectoría de este campus empezó a realizar acciones para restringir el acceso a personas ajenas a la comunidad universitaria, y segunda, porque la implementación de clases a distancia ha impedido que los estudiantes estén cotidianamente expuestos a los hurtos, robos y asaltos en las inmediaciones o en los trayectos del transporte público hacia o desde el campus.

No sorprende que alrededor de $40 \%$ de los alumnos haya sido víctima de la comisión de delitos en sus inmediaciones, porque los datos indican que se está perdiendo 
la gobernabilidad o no se está haciendo cumplir con la normatividad en las zonas de asentamiento: más del 60\% de los alumnos de ambas unidades académicas dijo que sabía que existían al menos cinco bares, cantinas o pulquerías a menos de 300 metros de las instalaciones escolares, lo cual está prohibido por la Ley de Establecimientos Mercantiles del 2018 (Congreso de la Ciudad de México, 2018). Las autoridades de las alcaldías donde se asientan los campus estudiados necesitan emprender acciones firmes para afianzar la gobernabilidad de sus territorios e imponer el cumplimiento de esta norma.

La recuperación de la gobernabilidad es importante para mejorar la vida académica de los campus universitarios porque, como esta investigación sugiere, entre $60 \%$ y $70 \%$ de los alumnos que son víctimas de actos de inseguridad sufren también de alguna consecuencia académica. Es posible que la más perniciosa, dejar de asistir a la universidad, ocurra en el 11\% de quienes han sido víctimas de un acto delictivo. Este dato no es menor, porque podría implicar que alrededor de 5 puntos porcentuales, de la reducida eficiencia terminal, se relacionen con los problemas de inseguridad interna y externa al campus, aunque esto es algo que requiere investigación más detallada, porque hace falta precisar la proporción del abandono que fue definitiva.

Las problemáticas ilustradas por la UAM, sobre todo la UAMI, relacionadas con las consecuencias académicas de la inseguridad interna y externa a los campus, y dado el actual contexto nacional de aumento en la criminalidad, llevan a retomar las sugerencias de los expertos en Crime Prevention Through Environmental Design (Deutsch, 2019) y de otros investigadores (Njoki, 2018; Díaz-Vicario y Gairín, 2017) para pugnar por que las universidades nacionales emprendan acciones, tanto de gestión interna como de coordinación externa, encaminadas a proteger sus instituciones de la criminalidad exterior. Internamente deben tomar en cuenta que el diseño y la disposición de las edificaciones pueden contribuir al mejoramiento de la vigilancia natural de los espacios y los accesos, y que el establecimiento de controles en las entradas mejora las condiciones objetivas de seguridad. Externamente, conviene a las rectorías universitarias coordinarse con los gobiernos locales, a fin de propiciar el cumplimiento de las normas jurídicas que inciden en la habitabilidad educativa de sus instituciones. Con ello las universidades evitarán que sus comunidades asuman la responsabilidad de enfrentar los problemas de inseguridad interior y exterior.

Es encomiable que muy recientemente, en febrero del 2020, el campus Iztapalapa logró establecer controles en los accesos peatonales principales, mediante torniquetes electrónicos. Esta acción implica un avance importante en la recuperación de la gobernabilidad del campus, y ojalá que sus comunidades estudiantiles, académicas y de trabajadores administrativos la refuercen, por sobre los intereses de grupos estudiantiles (UAMI-Consejo Iztapalapa, 2020) y sindicales (Nava, 2020). Hacemos votos porque las autoridades también logren avances en un plan integral de seguridad 
interior, que prevea la regulación del conocido "tianguis" asentado en su interior (Nava, 2020), porque podría ser aprovechado como vía de acceso furtivo para el narcomenudeo que amenaza al campus (Rubiera, 2020).

Esta investigación avanzó en explorar las consecuencias de la inseguridad universitaria interna y externa, recurriendo al muestreo por cuotas. La importancia del fenómeno, entendido como uno de los componentes centrales de la habitabilidad educativa universitaria, amerita la realización de investigaciones con técnicas de muestreo probabilístico, las cuales, aunque son más costosas, permiten la realización de estimaciones finales confiables, con técnicas de estadística inferencial. Ello permitiría valorar la significancia estadística de las pequeñas diferencias que en ocasiones se encontraron al contrastar los dos campus estudiados. Ojalá que en el futuro sea posible disponer de los recursos necesarios para poder estimar con mayor precisión la evolución de la inseguridad en la institución, a la luz de las acciones de gobernabilidad que se están empezando a realizar.

\section{REFERENCIAS}

ANUIES [Asociación Nacional de Universidades e Instituciones de Educación Superior] (2011). Manual de seguridad para instituciones de educación superior. Estrategias para la prevención y atención. ANUIES.

Cohen, J. (2006). Social, emotional, ethical and academic education: Creating a climate for learning, participation in democracy and well-being. Harvard Educational Review, 76(2), 201-237.

Cedillo, J. (2015, 4 abr.). Frontera norte. Éxodo estudiantil por la narcoviolencia. Proceso. Recuperado de: https:// www.proceso.com.mx/400252/frontera-norteexodo-estudiantil-por-la-narcoviolencia.

Côté-Lussier, C., y Fitzpatrick, C. (2016). Feelings of safety at school, socioemotional functioning, and classroom engagement. Journal of Adolescent Health, 58(5), 543-550. http://dx.doi.org/10.1016/j.jadohealth.2016.01.003.

Congreso de la Ciudad de México (2018, 4 may.). Ley de Establecimientos Mercantiles del Distrito Federal. Gaceta Oficial del Distrito Federal. https: / / www.congresocdmx.gob.mx/leyes-expedidas/.

Butkus, S. (2020). Investigating school shootings from 1996 to 2019 for processes needed to prevent and respond to future school shootings: A case study (Publication No. 2391983971) [Tesis doctoral]. Northcentral University. Recuperado de: https://search.proquest.com/ docview / 2391983971?pq-origsite=gscholar\&fromo penview $=$ true.

Dávila, P. (2018, 4 mar.). Indignación en la UNAM Graue, por replantear la política sobre las drogas. Proceso, (2157). Recuperado de: https://www.magzter.com/ article/News/Revista-Proceso/Indignacin-en-laUNAM-Graue-por-replantear-la-poltica-sobre-lasdrogas.

Deutsch, W. (2019). Crime prevention through environmental design. Recuperado de: https://www.thebalancesmb. $\mathrm{com} /$ crime-prevention-through-environmentaldesign-394571\#natural-surveillance.

Díaz-Vicario, A., y Gairín, J. (2017). A comprehensive approach to managing school safety: Case studies in Catalonia, Spain. Educational Research, 59(1), 89-106. http://dx.doi.org/10.1080/00131881.2016.1272430.

Dubet, F. (2003). Las figuras de la violencia en la escuela. Docencia, (19), 27-39.

Edmonton Police Service (2018). Creating safer communities through well-planned environmental design. Recuperado de: https://www.edmontonpolice.ca/CrimePrevention/ CommunitySafety/CPTED.

Erichsen, K. (2020). The influence of security and support on performance, punishment, and parental engagement in majority white and majority minority schools (Publication No. 27741928) [Tesis doctoral]. Florida State University. 
Recuperado de: https://search.proquest.com/doc view/2434382596/23E880DEA9EC4A73PQ/2?a ccountid $=37347$.

Fierro, C. (2017). Escuelas y docentes en contextos de violencia y exclusión. Contribución a la construcción de tejido social. En COMIE, Actas del Consejo Mexicano de Investigación Educativa (pp. 126-147). Consejo Mexicano de Investigación Educativa. Recuperado de: http://www.comie.org.mx/congreso/actas/.

Fritz, C. (2003). The learning environment as place: An analysis of the United States Department of Education's six design principles for learning environments. Washington State University. Recuperado de: https:/ / research.libraries. wsu.edu/xmlui/handle/2376/110.

Garnica, A. (2012). La seguridad en instituciones de educación superior. Estado actual y recomendaciones. ANUIES.

González, E., Inzunsa, P., y Benítez, L. (2013). Narcoviolencia en las escuelas. En A. Furlán y T. Spitzer. Convivencia, disciplina y violencia en las escuelas 2002-2011 (pp. 437-520). ANUIES/COMIE.

Hernández, J. M. (2010). Habitabilidad educativa de las escuelas. Marco de referencia para el diseño de indicadores. Sinéctica, (35), 241-254.

Imagen Radio (2020, 3 feb.). Situación en la UNAM, el reflejo de lo que pasa en el país, con Mario Luis Fuentes. Recuperado de: https://www.youtube.com/watch? $\mathrm{v}=\mathrm{TcOdgKwQzbM \& lc}=\mathrm{Ugzk} 0 \mathrm{AlKNKqJwKuTK}$ K54AaABAg.

Kennedy, M. (2017). Enhancing school security. American School \& University, 90(2), 14-17. Recuperado de: http://www.bidi.uam.mx:8331/login?url=https:// www.proquest.com/docview/1961351029?accoun tid $=37347$.

Marván, M. (2018). Violencia política y narcoviolencia. Hechos y derechos, (44). Recuperado de: https:// revistas.juridicas.unam. $\mathrm{mx} /$ index.php/hechos-yderechos/article/view/12173/13848.

Mayorga, F., y Córdova, E. (2007). Gobernabilidady gobernanza en América Latina. Recuperado de: http:/ /www. institut-gouvernance.org/docs/ficha-gobernabilida. pdf.

Nava, T. (2020, 17 ene.). Sesión de Consejo Académico 460 UAMI [UAMI-Consejo Iztapalapa]. Recuperado de: https://www.youtube.com/ watch? $\mathrm{v}=\mathrm{KTs}$ TwNBDrN4 (1h28m50s-1h31m30s).
Njoki, A. (2018). School safety and its influence on teaching and learning processes in public secondary schools in Nairobi and Nyeri counties, Kenya [Tesis doctoral no publicada]. Kenyatta University. Recuperado de: http://irlibrary. ku.ac.ke/bitstream/handle/123456789/18651/ School $\% 20$ safety $\% 20$ and $\% 20$ its $\% 20$ influence $\% 20$ on $\% 20$ teaching $\% 20$ and $\% 20$ learning $\% 20$ processes.... pdf? sequence $=1$.

Notimex (2012, 28 feb.). Homicidio de catedrático de la UAM, por quitarle 25 mil pesos. Excélsior. Recuperado de: http://www.excelsior.com.mx/2012/02/28/ comunidad/814364.

Ostrom, E. (2014). Más allá de los mercados y los Estados: gobernanza policéntrica de sistemas económicos complejos. Revista Mexicana de Sociología, 76(núm. especial), 15-70.

Poy, L. (2012, 10 mar.). Desecha la UNAM propuesta de seguridad de la ANUIES. La Jornada. Recuperado de: http://www.jornada.unam.mx/2012/03/10/ sociedad/032n1 soc.

Ramos, M., y Vázquez, R. (2013). El consumo y abuso de drogas, su relación con la violencia escolar y los programas preventivos. En A. Furlán y T. Spitzer (coords.). Convivencia, disciplina y violencia en las escuelas 2002-2011 (pp. 381-436). ANUIES/COMIE.

Redacción Proceso (2012, 28 feb.). Asesinan a profesor de la UAM; impartía clases sobre democracia y violencia. Proceso. Recuperado de: http://www.proceso.com. $\mathrm{mx} / 299561$ /asesinan-a-profesor-de-la-uam-impartiaclases-sobre-democracia-y-violencia.

Rubiera, G. (2020, 14 ene.). Narcomenudeo, inseguridad y violencia en la UAM Iztapalapa [La Octava]. Recuperado de: https://www.youtube.com/ watch? $\mathrm{v}=\mathrm{Nfues} W 8 j \mathrm{jtE} \& \mathrm{t}=244 \mathrm{~s}$.

Sohn, D-W. (2015). Residential crimes and neighbourhood built environment: Assessing the effectiveness of crime prevention through environmental design (CPTED). Cities, 52(2016), 86-93. Recuperado de: https://scihub.tw/https://doi.org/10.1016/j.cities.2015.11.023.

Tanner-Smith, E., Fisher, B., Addington, L., y Gardella, J. (2017). Adding security, but subtracting safety? Exploring schools' use of multiple visible security measures. Am J Crim Just, (43), 102-119.

Thapa, A., Cohen, J., Guffey, S., y Higgins-D’Alessandro, A. (2013). A review of school climate research. Recu- 
perado de: http://rer.sagepub.com/content/ear ly/2013/04/18/0034654313483907.

Times Higher Education (2019). World University Rankings 2020. Recuperado de: https://www.timeshighereducation.com/world-university-rankings/2020/world-ranking\#!/page/0/length/-1/sort_by/rank/sort_order/ asc/cols/stats.

UAM (2019). Anexo estadístico del informe del Rector General 2018. Rectoría General de la Universidad Autónoma Metropolitana. Recuperado de: http://www.uam.mx/ informesrg/.

UAMI (2017a, 24 feb.). iLa seguridad la hacemos todos! Comunicado a la comunidad universitaria. Rectoría de la Unidad Iztapalapa.

UAMI (2017b, 27 feb.). Aviso a la comunidad universitaria. Rectoría de la Unidad Iztapalapa.
UAMI (2017c, 1 mar.). ¡No te dejes sorprender! iNo entregues tus pertenencias, bajo ninguna circunstancia! Aviso a la comunidad universitaria. Rectoría de la Unidad Iztapalapa.

UAMI-Consejo Iztapalapa (2020, 14 feb.). Mesa de diálogo en la UAMI. Recuperado de: https:/ / www.youtube.

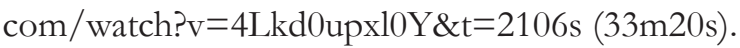

UNODC (2019). Global study on homicide, 2019. United Nations Publications. Recuperado de: https://www. unodc.org/unodc/en/data-and-analysis/globalstudy-on-homicide.html.

Villalobos, A. (2018, 4 mar.). En CU, hasta el personal de seguridad está en riesgo. Proceso, (2157). Recuperado de: https://www.proceso.com.mx/525007/ en-cu-hasta-el-personal-de-seguridad-esta-enriesgo.

Cómo citar este artículo:

Hernández Vázquez, J. M., Rodríguez Lagunas, J., y Leyva Piña, M. A. (2021). Inseguridad escolar y problemas académicos en una universidad pública mexicana. IE Revista de Investigación Educativa de la REDIECH, 12, e1114. doi: 10.33010/ie_rie_rediech.v12i0.1114. 